\title{
On the geometric phase in the spatial equilibria of nonlinear rods
}

\author{
Peinan Zhong ${ }^{1,2}$ - Guojun Huang ${ }^{1,2}$ - Guowei Yang ${ }^{1,2}$
}

Received: 10 May 2016 / Revised: 7 October 2016 / Accepted: 25 October 2016 / Published online: 20 January 2017

(C) The Chinese Society of Theoretical and Applied Mechanics; Institute of Mechanics, Chinese Academy of Sciences and Springer-Verlag Berlin Heidelberg 2017

\begin{abstract}
Geometric phases have natural manifestations in large deformations of geometrically exact rods. The primary concerns of this article are the physical implications and observable consequences of geometric phases arising from the deformed patterns exhibited by a rod subjected to end moments. This mechanical problem is classical and has a long tradition dating back to Kirchhoff. However, the perspective from geometric phases seems to go more deeply into relations between local strain states and global geometry of shapes, and infuses genuinely new insights and better understanding, which enable one to describe this kind of deformation in a neat and elegant way. On the other hand, visual representations of these deformations provide beautiful illustrations of geometric phases and render the meaning of the abstract concept of holonomy more direct and transparent.
\end{abstract}

Keywords Geometric exact rod - Geometric phase . Rotation group $\cdot$ Kirchhoff analogy

\section{Introduction}

Since Berry's introduction of the adiabatic geometrical phase, a large amount of research has appeared on the theoretical foundations, physical applications, and experimental manifestations of geometric phases. In quantum mechanics,

$\triangle$ Guowei Yang

gwyang@imech.ac.cn

$1 \quad$ Key Laboratory for Mechanics in Fluid Solid Coupling Systems, Institute of Mechanics, Chinese Academy of Sciences, Beijing 100190, China

2 School of Engineering Science, University of Chinese Academy of Science, Beijing 100049, China
Berry [1] has first shown that when the Hamiltonian $H(\lambda)$, which depends on a set of parameters $\lambda=\left(\lambda_{1}, \lambda_{2}, \ldots\right)$, undergoes adiabatic evolution along a closed curve $\gamma$ in the parameter space, then the state vector corresponding to this quantum system evolves into a vector which agrees with the initial vector only up to a phase factor $\mathrm{e}^{\mathrm{i} \phi}$. This phase $\phi$ can have observable consequences, and contains, in addition to the usual dynamics phase, a purely geometric part, which depends only on the geometry of the closed curve $\gamma$. Soon after this discovery, Hannay [2] and Berry [3] have shown that, under a closed adiabatic loop in the space of classically integrable Hamiltonians, the angle variables pick up extra phases in addition to the time integral of the instantaneous frequencies. This classical analogue of the Berry's quantum phase, called Hannay angles, can be explained by the fact that the action-angle variables $(\boldsymbol{I}, \boldsymbol{\varphi})$ are parameter-dependent so that canonical transformation to these coordinates produces an additional term in the Hamiltonian.

The fact that the geometric phase has important observable consequences in physics is not the only reason why it has attracted so much attention. The geometric phase is also one of the most beautiful examples of what Wigner once called the unreasonable effectiveness of mathematics in the natural science. In geometry, when an orthonormal frame returns after traversing a closed path to its original position, but rotated, the rotation is referred to as holonomy. This is a unifying mathematical concept that underlies the occurrence of geometric phases in natural phenomena. In the quantum case, immediately after Berry's introduction of the adiabatic geometric phase, Berry [1,3] and Simon [4] noticed that it could be interpreted as the holonomy of a fiber bundle and that Berry's gauge potential played the role of a connection on this fiber bundle. For the classical adiabatic angles, Golin et al. [5] and Montgometry [6] defined an 
Ehresmann connection by averaging, which they called the Hannay-Berry connection, and have shown that the Hannay angles are the holonomy of this connection on a Poisson fiber bundle associated with the given problem. In fact, the study of geometric phases can be put into the framework of connections on bundles and reduction of Hamiltonian systems with symmetry. Various other holonomy phenomena can be shown to be instances of the reconstruction procedure for the dynamics of a given Hamiltonian system from the induced system on the reduced phase space. It was this relation to the beautiful mathematics of fiber bundles and symmetry that caused the geometric phase to become a fashion in mathematical physics. A detailed presentation of reduction and reconstruction can be found in Marsden [7] and Marsden et al. [8].

The idea of geometric phases, like group theory, has now become part of the lingua franca in quantum mechanics, with applications in fields ranging from chemistry to condensed matter physics. Yet its inherent universality has not been widely appreciated in the theory of elasticity. The present work is our initial attempt to unravel effects of geometric phases arising in the nonlinear rod theory. To do this, we first concentrate on the equilibrium states exhibited by an arbitrarily long rod, deformed by external moments applied at one of its ends. The physical model we consider here is a simple boundary value problem; however, the deformed patterns produced by it are rather complex. To understand the situations here, we exploit the Kirchhoff kinetic analogy: the equations governing equilibrium states are formally equivalent to the Euler equations describing the motion of rigid body pivoted at a fixed point (see Love [9], more modern treatment can be found in Refs. [10,11]). This remarkable feature immediately implies that the distribution of a stress field is periodic. During a period of stress, the configuration acquires a rotation around the direction of external moment with a nontrivial angle $\alpha^{*}$, a manifestation of holonomy, and this is the phenomenon of interest here. The underlying geometry of this phase can be understood in terms of the rigid-body phase [8], and its physical implications on the deformed configuration allow us to break the rod down to find its underlying parts. Then the whole deformed rod can be build up through affine transformation of each individual part. In fact, this kind of description can be considered as the generalization of the Poinsot's description of the rigid-body motion to the larger deformations of rods. Therefore, when this problem that is not ordinarily associated with geometric phases is phased in terms of them, the result we arrive at is a clear understanding of the structure of this problem, and an elegant expression of its solution. On the other hand, the physical consequences of geometric phases on rods reveal the abstract topological concept holonomy through vivid and intuitive visual deformation shapes.
The corresponding rigid-body phase formula based on the theory of connections and the formula for holonomy in terms of curvature was given by Montgomery [12] and Marsden et al. [8]. Its interesting history and practical applications in control theory can be referred to in Ref. [13] and references therein.

The concept that has emerged as central is the geometric phase, which is subtle and can be difficult to grasp on the first encounter. The simple model studied here help us to demystify it by describing how it lies at the heart of beautiful patterns exhibited by the rod deformation. With the increasing separation between daily experience and the more theoretical branches of physics, it would be comforting to understand phenomena that contains deep physical ideas and leads to effects which can be readily realized.

This paper is organized as follows. In Sect. 2, we outline the basic geometric exact rod theory. The main concerns of this paper are given in Sect. 3. First, in this part, the occurrence and consequences of geometric phases in the spatial shapes exhibited by rods are analyzed in detail. The phase $\alpha^{*}$ arising here, is analogous to cyclic adiabatic evolutions of quantum systems, and separates naturally into the obvious dynamical change (the integral of the strain component along the direction of end moment), and an additional geometric change (the solid angle subtended by a curve traced on a sphere by the direction of the material moment). Section 4 is devoted to elaborate theoretical and numerical factors on how observable consequences of the geometric phases might be extracted from deformed shapes of rods. In Appendix, the phase formula is ascertained mathematically by an argument similar to the one taken up by Montgomery [12], but the details in this proof have been improved considerably in order to obtain more geometric insights into this relation.

\section{Geometric exact rod theory}

We come to a brief exposition of the fundamental mathematical formalism of this nonlinear rod model. Only those aspects of the theory relevant to the subsequent developments are addressed. The reader could refer to Simo [14] and Simo et al. [15] for further mathematical and physical contents of the geometric exact rod model.

Let $\left\{\boldsymbol{e}_{i}\right\}_{i=1,2,3}$ be the orthonormal basis vectors in a inertial frame with origin $O$. We consider a rod with initial length $L$. The arc-length parameter $s$ for the center-line is employed as a material coordinate, i.e., a parameter whose value at a material point is constant in time, which we take to vary over the interval $[0, L]$. A plane cross-section $\mathscr{A}(s)$ of the rod, initially perpendicular to the line of centroids, is assumed to remain plane after deformation, but, taking account of shear deformation, needs not be perpendicular to the deformed center-line. 
Let an orthonormal frame $\left\{\boldsymbol{d}_{1}, \boldsymbol{d}_{2}, \boldsymbol{d}_{3}\right\}$ attached to a typical section with origin placed at the centroid be chosen such that $\boldsymbol{d}_{1}$ and $\boldsymbol{d}_{2}$ are directed along the principal axes of the section and $\boldsymbol{d}_{3}$ is normal to the cross section $\mathscr{A}$ at all times. A simplification of the model comes from the assumption that $\boldsymbol{d}_{3}$ is tangent to the center-line at reference configuration. Motivated by a terminology widely used in rigid body mechanics, $\left\{\boldsymbol{d}_{i}\right\}_{i=1,2,3}$ should be referred as the body frame.

The basis of the mathematical formalism of geometric exact rod theory lies in the fact that any configuration can be described, at a given moment, by a definite map

$\Phi: s \in[0, L] \mapsto(\varphi(s), \boldsymbol{\Lambda}(s)) \in \mathbb{R}^{3} \times \mathrm{SO}(3)$,

with the vector function $\varphi(s)$ indicating the current position of the line of centroids and the orthogonal matrix field $\boldsymbol{\Lambda}(s)$ specifying the orientation of the body frame relative to the fixed basis $\left\{\boldsymbol{e}_{i}\right\}_{i=1,2,3}$ such that

$\boldsymbol{d}_{i}=\boldsymbol{\Lambda} \boldsymbol{e}_{i}$

Formally, the abstract configuration space of a rod is the infinite dimensional non-linear manifold

$\mathscr{Q}:=\left\{\Phi \triangleq(\boldsymbol{\varphi}, \boldsymbol{\Lambda}):[0, L] \mapsto \mathbb{R}^{3} \times \mathrm{SO}(3)\right\}$.

As a convention, the reference configuration is denoted by $\Phi_{0}=\left(\boldsymbol{\varphi}_{0}, \boldsymbol{\Lambda}_{0}\right)$.

The geometry of a deformed rod is encapsulated in the frame invariant material strain measures

$\boldsymbol{\Gamma}=\boldsymbol{\Lambda}^{\mathrm{T}} \boldsymbol{\varphi}^{\prime}-\boldsymbol{\Lambda}_{0}^{\mathrm{T}} \boldsymbol{\varphi}_{0}^{\prime}$ and $\hat{\boldsymbol{\Omega}}=\boldsymbol{\Lambda}^{\mathrm{T}} \boldsymbol{\Lambda}^{\prime}-\boldsymbol{\Lambda}_{0}^{\mathrm{T}} \boldsymbol{\Lambda}_{0}^{\prime}$

From now on, primes $(\cdot)^{\prime}$ are used to mark the spatial derivatives along the center-line, that is with respect to the arc-length parameter $s$. Here, a superposed hat $(\hat{.})$ denotes the skew-symmetric matrix (in $\mathfrak{s o}(3)$ ) associated with a vector (in $\mathbb{R}^{3}$ ). In matrix notation, we have the relations

$\boldsymbol{\xi}=\left[\begin{array}{l}\xi_{1} \\ \xi_{2} \\ \xi_{3}\end{array}\right] \mapsto \hat{\boldsymbol{\xi}}=\left[\begin{array}{ccc}0 & -\xi_{3} & \xi_{2} \\ \xi_{3} & 0 & -\xi_{1} \\ -\xi_{2} & \xi_{1} & 0\end{array}\right]$,

which follows from the intrinsic definition

$\hat{\xi} a=\xi \times a$ for all $a \in \mathbb{R}^{3}$.

Physically, $\Gamma_{1}$ and $\Gamma_{2}$ are shear in the $\boldsymbol{d}_{1}$ and $\boldsymbol{d}_{2}$ directions and $\Gamma_{3}$ is extension in the $\boldsymbol{d}_{3}$ direction; $\Omega_{1}$ and $\Omega_{2}$ represent bending in the $\left(\boldsymbol{d}_{2}, \boldsymbol{d}_{3}\right)$ and $\left(\boldsymbol{d}_{3}, \boldsymbol{d}_{1}\right)$ planes, respectively, and $\Omega_{3}$ is torsion of the rod, or the twist rate.

In what follows, for the purpose of this work, our attention is limited to rods that are naturally straight and untwisted in the undistorted and, hence, stress-free configurations. Then the body frame $\left\{\boldsymbol{d}_{k}\right\}_{k=1,2,3}$ can be chosen to be independent of $s$ and coincide with the standard basis in $\mathbb{R}^{3}$, i.e., $\boldsymbol{\Lambda}_{0}(s)$ equals to the $3 \times 3$ identity matrix $\boldsymbol{I}_{3}$. This immediately leads to $\boldsymbol{\Lambda}_{0}^{\mathrm{T}} \boldsymbol{\Lambda}_{0}^{\prime}=0$, and according to the definition rotational strain measure (4), one can write the derivative of $\boldsymbol{\Lambda}$ in terms of the vector $\boldsymbol{\Omega}$

$\Lambda^{\prime}=\boldsymbol{\Lambda} \hat{\boldsymbol{\Omega}}$.

As a direct consequence of it, the derivative of vector field $\boldsymbol{d}_{i}$ in the body frame may then take the form

$\boldsymbol{d}_{i}^{\prime}=\boldsymbol{\omega} \times \boldsymbol{d}_{i}$ for $i \in[1,2,3]$,

with

$\omega:=\Lambda \Omega=\Omega_{1} d_{1}+\Omega_{2} d_{2}+\Omega_{3} d_{3}$.

The vector $\omega(s)$ is the spatial rotational strain measure, signifying the rate of rotation per unit length along the rod. Following Kirchhoff and Love [9], the interpretation of Eq. (8) is that the body frame rotates with rotation velocity $\omega$, the arc-length $s$ along the center-line plays the role of time-like coordinates.

After the above geometric descriptions, we turn to the mechanics of this rod model. Stresses acting across the material cross-section $\mathscr{A}(s)$ are reduced to a resultant force $\boldsymbol{n}(s)$ and moment $\boldsymbol{m}(s)$ exerted on the side $s^{-}$by the material on the side $s^{+}$. Balancing of forces and moments for infinitesimal rod elements immediately yields the following coordinates free equilibrium equations

$$
\begin{aligned}
n^{\prime}+\bar{n} & =\mathbf{0}, \\
\boldsymbol{m}^{\prime}+\varphi^{\prime} \times \boldsymbol{n}+\overline{\boldsymbol{m}} & =\mathbf{0},
\end{aligned}
$$

where $\overline{\boldsymbol{n}}$ and $\overline{\boldsymbol{m}}$ are the prescribed forces and torque per unit of reference arc length.

In order to close the system (10), additional information relating the local forces and moments (stress) to the elastic deformations of the body (strain) need to be introduced. If the rod is made of hyperelastic material, there exists a scalar valued stored energy function $W\left(\Gamma_{i}, \Omega_{i}, s\right)$ characterizing the elastic property of the medium and dependent upon the strains and arc-length, with the property that the components of stress $N_{i}:=\boldsymbol{n} \cdot \boldsymbol{d}_{i}$ and $M_{i}:=\boldsymbol{M} \cdot \boldsymbol{d}_{i}$ satisfy the constitutive relations

$N_{i}=\frac{\partial W\left(\Gamma_{k}, \Omega_{k}\right)}{\partial \Gamma_{i}}$ and $M_{i}=\frac{\partial W\left(\Gamma_{k}, \Omega_{k}\right)}{\partial \Omega_{i}}$.

The triples $\boldsymbol{N} \equiv\left(N_{1}, N_{2}, N_{3}\right)$ and $\boldsymbol{M} \equiv\left(M_{1}, M_{2}, M_{3}\right)$ can also be interpreted as a material description of stress defined 
by pulling back the stress resultants $(\boldsymbol{n}, \boldsymbol{m})$ to the reference configuration with the orthogonal transformation $\boldsymbol{\Lambda}$

$\boldsymbol{N}=\boldsymbol{\Lambda}^{\mathrm{T}} \boldsymbol{n}$ and $\boldsymbol{M}=\boldsymbol{\Lambda}^{\mathrm{T}} \boldsymbol{m}$.

Traditionally, the components $M_{1}$ and $M_{2}$ of $\boldsymbol{M}$ are called the bending moments and $M_{3}$ the torsion moment, and components of $N$ are called the shear and extension forces.

A standard approximation in rod theory is to assume the body to be linear elastic, then the stored energy function is provided by the quadratic form

$W(\boldsymbol{\Gamma}, \boldsymbol{K})=\frac{1}{2} \boldsymbol{\Gamma} \cdot \boldsymbol{C}_{N} \boldsymbol{\Gamma}+\frac{1}{2} \boldsymbol{\Omega} \cdot \boldsymbol{C}_{\boldsymbol{M}} \boldsymbol{\Omega}$,

with

$\boldsymbol{C}_{\boldsymbol{N}}=\operatorname{diag}\left(G A_{1}, G A_{2}, E A\right)$ and $\boldsymbol{C}_{\boldsymbol{M}}=\operatorname{diag}\left(E I_{1}, E I_{2}, G J\right)$,

where $G A_{1}$ and $G A_{2}$ denote, respectively, the shear stiffness along the axes $\boldsymbol{d}_{1}$ and $\boldsymbol{d}_{2}, E A$ is the axial stiffness along $\boldsymbol{d}_{3}$, $E I_{1}$ and $E I_{2}$ are the principal bending stiffness relative to axes $\boldsymbol{d}_{1}$ and $\boldsymbol{d}_{2}$, respectively, and $G J$ is the Saint-Venant torsional stiffness along $\boldsymbol{d}_{3}$. The constitutive relations can be expressed in terms of linear equations

$N=C_{N} \Gamma, \quad M=C_{M} \Omega$.

From this point on, we assume that the rod is made of linear elastic material and has uniform properties, both geometrical and mechanical, along its length. Mathematically, by virtue of this homogeneity, the stored energy function, and hence, the moduli (constants of proportionality) occurring in the linear constitutive Eq. (13) do not vary along the rod.

This completes the basic theory of rod needed for subsequent developments.

\section{Geometric phase}

The main concern of this paper arises from a particular boundary value problem of Eq. (10). Let us now consider a rod with straight reference configurations clamped at one end $(s=0)$ and free at the other $(s=L)$,

$\varphi(0)=\mathbf{0}$ and $\boldsymbol{\Lambda}(0)=\tilde{\boldsymbol{\Lambda}}$.

Both applied distributed force and moment vanish, $\overline{\boldsymbol{n}}=\overline{\boldsymbol{m}}=$ 0. The only external loads are couples applied at the free end and enter the problem as boundary conditions,

$\boldsymbol{n}(L)=\mathbf{0}$ and $\boldsymbol{m}(L)=\tilde{\boldsymbol{m}}$.
In what follows, we shall investigate the deformation of the rod under such conditions through geometric reasoning without the necessity of actually solving the differential equation. It will become clear that the complexity of rod shapes of spatial equilibrium states is fundamentally of geometric origin.

The equilibrium Eq. (10) for rods loaded only at their ends are simply expressed in terms of the internal force and moment vectors:

$\boldsymbol{n}=$ const,$\quad \boldsymbol{m}^{\prime}+\boldsymbol{\varphi}^{\prime} \times \boldsymbol{n}=\mathbf{0}$.

In view of the boundary condition (15), the contact force $\boldsymbol{n}$ vanishes: $\boldsymbol{n}=\mathbf{0}$. The linear constitutive equation of strain measure (4) yields $\boldsymbol{\Gamma}=\mathbf{0}$. Hence, the rod under consideration does not suffer extension and shear effect. This implies that a normal vector of each cross-section $\boldsymbol{d}_{3}$ is tangent to the center line and the parameter $s$ can also be interpreted as the arc-length along the rod in any deformed configuration. Therefore, taking account of the boundary condition (14), the position $\varphi$ of the center-line can be determined simply by the integral

$\boldsymbol{\varphi}=\int_{0}^{s} \boldsymbol{d}_{3} \mathrm{~d} s$.

Moreover, on account of $\boldsymbol{n}=\mathbf{0}$, the second equation in Eq. (16) yields the fact that the internal moment $\boldsymbol{m}$ is a constant vector along the center-line

$\boldsymbol{m}(s)=\tilde{\boldsymbol{m}}$ for all $s \in[0, L]$.

Since rotation leaves the space metric invariant, all the possible material moments $\boldsymbol{M}=\boldsymbol{\Lambda}^{\mathrm{T}} \boldsymbol{m}$ are vectors with constant norm $M:=\|\boldsymbol{m}\|$

$M_{1}^{2}+M_{2}^{2}+M_{3}^{2}=M^{2}$.

Therefore, the distribution of stresses along the rod becomes easier to understand, if we study it in a sphere $\mathscr{S}^{2}$ with radius $M$ through the map

$\phi_{m}: \mathrm{SO}(3) \mapsto \mathscr{S}^{2}, \quad \phi_{\boldsymbol{m}}: \boldsymbol{\Lambda} \mapsto \boldsymbol{\Lambda}^{\mathrm{T}} \boldsymbol{m}$.

Accordingly, when the vector $\boldsymbol{M}(s)$ varies along the centerline, its terminus moves along a curve on the sphere $s \mapsto$ $\boldsymbol{M}(s)=\boldsymbol{\Lambda}^{\mathrm{T}}(s) \boldsymbol{m} \in \mathscr{S}^{2}$. After taking the derivative of $\boldsymbol{M}(s)$, and with the help of Eq. (7), we find

$\boldsymbol{M}^{\prime}=(\boldsymbol{\Lambda} \hat{\boldsymbol{\Omega}})^{\mathrm{T}} \boldsymbol{m}=-\hat{\boldsymbol{\Omega}} \boldsymbol{M}$.

This relation clearly has the same form of the famous Euler equation for the free motion of a rigid body (see Arnol'd [16]) 
$M^{\prime}=\boldsymbol{M} \times \boldsymbol{\Omega}$.

Further simplification comes from the observation that $W(\boldsymbol{\Gamma}, \boldsymbol{\Omega})$, the elastic energy function per unit length of the rod is another conserved quantity of the system. In our case, the function $W$ just depends on the strain $\boldsymbol{\Omega}$ vector, or equivalently, the stress $\boldsymbol{M}$

$W=\frac{1}{2} \boldsymbol{\Omega} \cdot \boldsymbol{C}_{\boldsymbol{M}} \boldsymbol{\Omega}=\frac{1}{2} \boldsymbol{M} \cdot \boldsymbol{C}_{\boldsymbol{M}}^{-1} \boldsymbol{M}$.

By taking the derivative of $W$ along the center line, we obtain

$W^{\prime}=\boldsymbol{\Omega} \cdot \boldsymbol{M}^{\prime}=\boldsymbol{\Omega} \cdot(\boldsymbol{M} \times \boldsymbol{\Omega})=0$.

Hence, the quantity $W$ keeps constant, and its value can be determined at position $s=0$

$W=\left.\frac{1}{2} \boldsymbol{m} \cdot\left(\boldsymbol{\Lambda} \boldsymbol{C}_{\boldsymbol{M}}^{-1} \boldsymbol{\Lambda}^{\mathrm{T}}\right)\right|_{s=0} \boldsymbol{m}$.

The conservation of $W$ restricts the vector field $\boldsymbol{M}(s)$ lying on the intersection of the sphere defined by Eq. (19) with the ellipsoid given by

$\frac{M_{1}^{2}}{E I_{1}}+\frac{M_{2}^{2}}{E I_{2}}+\frac{M_{3}^{2}}{G J}=2 W$.

From these geometric considerations, we can already draw some conclusions concerning the deformation. First of all, we notice that, for a typical value of $W$, the orbit of moment vector $\boldsymbol{M}$ along the center line of the rod describes a closed curve on the sphere $\mathscr{S}^{2}$. The distribution of $\boldsymbol{M}$ must be periodic; during one period $S_{\sigma}$, it returns to its original position

$\boldsymbol{M}\left(s+S_{\sigma}\right)=\boldsymbol{M}(s)$.

We call this period $S_{\sigma}$ the stress-period.

Next, we study the change of rotation field after one stress period $S_{\sigma}$. Generally, it can not be expected that the rotation field will repeat itself. In fact, the periodic variation of $\boldsymbol{M}$ leads to the following relation

$\boldsymbol{m}=\boldsymbol{R} \boldsymbol{m}, \quad$ where $\boldsymbol{R}:=\boldsymbol{\Lambda}\left(s+S_{\sigma}\right) \boldsymbol{\Lambda}^{\mathrm{T}}(s)$.

From the above equation, we can infer that the matrix $\boldsymbol{R}$ is a rotation about the direction of $\boldsymbol{m}$ through an angle $\alpha^{*}$

$\boldsymbol{R}=\exp \left(\alpha^{*} \boldsymbol{\mu}\right)$

where the map exp : $\mathbb{R}^{3} \mapsto \mathrm{SO}(3)$ is the exponential map defined on $\mathrm{SO}(3)$ group, and $\boldsymbol{\mu}=\boldsymbol{m} / M$ is the normalization of the spatial moment $\boldsymbol{m}$.

The physical consequence of Eqs. (25) and (27) can be understood as follows. Let the configuration of a rod be given by $\Phi \in \mathscr{Q}$. We divide this rod into several segments and each of them is contained in a range

$I_{k}=\left[(k-1) S_{\sigma}, k S_{\sigma}\right], \quad k=1,2, \ldots, n$.

The configuration $\Phi_{[k]}$ of $k$-th segment is the restriction of $\Phi$ on the sub-interval $I_{k}$ such that

$\Phi_{[k]}(t)=\Phi\left((k-1) S_{\sigma}+t\right)$ for $t \in\left[0, S_{\sigma}\right]$.

According to Eq. (25), all of these segments share identical distribution of stress $\boldsymbol{M}$, or of the strain $\boldsymbol{\Omega}$. It is evident from physics arguments that each segment must exhibit a same deformation, since in the local coordinate system (given by the moving frame), the same internal stress is exerted on their corresponding rod elements.

The same conclusion may also be drawn mathematically. According to the classical theory of differential geometry, the shape of center lines is determined by the curvature and torsion function $\kappa, \tau_{g}$ (see Spivak [17]). However, they can be directly related to the strain vector $\boldsymbol{\Omega}$. To demonstrate this, we need first to understand the relation between the Serret-Frenet Frame $\{\boldsymbol{n}, \boldsymbol{b}, \boldsymbol{t}\}$ and the directors $\boldsymbol{d}_{i}$. As we have mentioned earlier, the tangent vector $\boldsymbol{t}$ is to coincide with $\boldsymbol{d}_{3}$, and it is evident that the principal normal $\boldsymbol{n}$ and binormal $\boldsymbol{b}$ lie on the $\boldsymbol{d}_{1}, \boldsymbol{d}_{2}$ plane. Since

$\boldsymbol{t}^{\prime}=\boldsymbol{\Omega} \times \boldsymbol{t}=\Omega_{2} \boldsymbol{d}_{1}-\Omega_{1} \boldsymbol{d}_{2}$,

from the Serret-Frenet equation $\boldsymbol{t}^{\prime}=\kappa \boldsymbol{n}$, we have

$\kappa=\sqrt{\Omega_{1}^{2}+\Omega_{2}^{2}}, \quad \boldsymbol{n}=\cos (\psi) \boldsymbol{d}_{1}+\sin (\psi) \boldsymbol{d}_{2}$,

where the angle $\psi$ is introduced via

$\left(\Omega_{1}, \Omega_{2}\right)=(-\kappa \sin \psi, \kappa \cos \psi)$.

Taking account of the definition $\boldsymbol{b}:=\boldsymbol{t} \times \boldsymbol{n}$, the binormal $\boldsymbol{b}$ can also be expressed in terms of $\left(\boldsymbol{d}_{1}, \boldsymbol{d}_{2}\right)$

$\boldsymbol{b}=-\sin (\psi) \boldsymbol{d}_{1}+\cos (\psi) \boldsymbol{d}_{2}$.

With relations (29) and (31) in hand, we notice that the strain $\omega$ takes the form

$\omega=\kappa b+\tau t$,

where $\tau:=\Omega_{3}$ is the elastic twist. By using this expression, after taking the derivative of $\boldsymbol{b}$ in Eq. (31), we observe that

$\boldsymbol{b}^{\prime}=-\psi^{\prime} \boldsymbol{n}+\boldsymbol{\omega} \times \boldsymbol{b}=-\left(\psi^{\prime}+\tau\right) \boldsymbol{n}$. 
In view of the definition $\tau_{g}$ given by the Serret-Frenet equation $\boldsymbol{b}^{\prime}=-\tau_{g} \boldsymbol{n}$, the geometric torsion function can be written as the sum

$\tau_{g}=\psi^{\prime}+\tau$,

where the derivative $\psi^{\prime}$ is called the twist of Love [9]. Finally, we can conclude that the local geometry of the center-line is totally described by the strain vector $\boldsymbol{\Omega}$. Since each segment has a same distribution of $\boldsymbol{\Omega}$, they must share an identical curvature and torsion function $\kappa$ and $\tau_{g}$. Accordingly, a cyclical pattern has taken shape in the center-line as a result of this periodical variation of the strain vector $\boldsymbol{\Omega}$.

Let us ascertain the relation between configuration $\Phi_{[1]}$ and $\Phi_{[2]}$. For the rotational part, it is evidently from Eq. (27) that

$\boldsymbol{\Lambda}_{[2]}=\boldsymbol{R} \boldsymbol{\Lambda}_{[1]}$.

With help of Eqs. (17) and (28), we find that

$\boldsymbol{\varphi}_{[2]}(t)=\int_{S_{\sigma}}^{t+S_{\sigma}} \boldsymbol{d}_{3} \mathrm{~d} s+\int_{0}^{S_{\sigma}} \boldsymbol{d}_{3} \mathrm{~d} s=\boldsymbol{R} \boldsymbol{\varphi}_{[1]}(t)+\boldsymbol{u}$,

where the constant translation $\boldsymbol{u}$ is used to denote the position of the center-line defined by the integral

$\boldsymbol{\varphi}\left(S_{\sigma}\right)=\int_{0}^{S_{\sigma}} d_{3} \mathrm{~d} s$

Therefore, the configuration $\Phi_{[2]}$ can be obtained through the rigid motion consisting of the rotation $\boldsymbol{R}$ and the translation $u$

$\left(\varphi_{[2]}, \Lambda_{[2]}\right)=\left(\boldsymbol{R} \varphi_{[1]}+u, R \Lambda_{[1]}\right)$

This rule can be generalized at once to the case of $\Phi_{[k]}$ and $\Phi_{[k+1]}$

$\left(\boldsymbol{\varphi}_{[k+1]}, \boldsymbol{\Lambda}_{[k+1]}\right)=\left(\boldsymbol{R} \boldsymbol{\varphi}_{[k]}+\boldsymbol{u}, \boldsymbol{R} \boldsymbol{\Lambda}_{[k]}\right)$.

In consequence of this equation, we can obtain the configuration of $k$-th segment via $(k-1)$ times supposition of rigid motion $(\boldsymbol{R}, \boldsymbol{u})$ on the first segment. Abstractly, since $(\boldsymbol{R}, \boldsymbol{u})$ is an element of a special Euclidean group $\mathrm{SE}(3)$, we can conclude that

$\Phi_{[k]}=(\boldsymbol{R}, \boldsymbol{u})^{(k-1)} \cdot \Phi_{[1]}$,

where the power $(\boldsymbol{R}, \boldsymbol{u})^{(k-1)}$ is defined via the product of the $\mathrm{SE}(3)$ group

$(\boldsymbol{A}, \boldsymbol{a})(\boldsymbol{B}, \boldsymbol{b})=(\boldsymbol{A B}, \boldsymbol{A b}+\boldsymbol{a})$.
To sum up, these segments differed from each other by an affine transformation (a rigid-body motion), and the whole deformed pattern can be inferred from the knowledge of any one of them. It is interesting to compare this description of deformation with the Poinsot's description of the rigid-body motion (see Arnol'd [16]). The former could be considered as a generalization of the latter to the geometrically exact rod.

At this stage, it remains to determine the rotation $\boldsymbol{R}$ and the translation $\boldsymbol{u}$. For the rotation matrix $\boldsymbol{R}$, since its axis is already known in the direction of $\boldsymbol{m}$, the only thing remains to investigate is how much does it rotate.

It is remarkable that the angle $\alpha^{*}$ of the rotation $\boldsymbol{R}$ about the axis $\boldsymbol{m}$ after one stress period $S_{\sigma}$, can be expressed explicitly by the phase formula

$\alpha^{*}=\left(\frac{2 W S_{\sigma}}{M}-A\right) \bmod 2 \pi$.

The influence of topology on large deformations of a rod can be directly observed from this concise and informative expression. The part $2 W S_{\sigma} / M$ is called the dynamic phase, since it is totally determined by the mechanical property of this system, that is the energy per unit length $W$ and the magnitude $M$ of the moment $\boldsymbol{m}$. We notice that the value $2 W / M$ can be expressed in the form

$\frac{2 W}{M}=\omega \cdot \frac{m}{M}$

which can be interpreted as the rate of rotation about the direction of $\boldsymbol{m}$. Its occurrence in Eq. (40), as we expect, represents the local strain state of the rod. However, the nonobvious part is the signed solid angle $A$ enclosed by the closed orbit of the moment vector $\boldsymbol{M}$ along the rod, which clearly results from the global geometric property of the stress distribution. Hence, $A$ is called the geometric phase.

The beauty of geometry underlying the relation (40) could be efficiently appreciated in terms of reduction and reconstruction (see Masden and Weinstein [18] and Masden [7] for more detailed treatments). First, we can picture the rotation and material moment fields as tracing out a path in the cotangent bundle $\mathscr{M}:=T^{*} \mathrm{SO}(3)$ of the rotation group:

$s \mapsto(\boldsymbol{\Lambda}(s), \boldsymbol{M}(s)) \in \mathscr{M}$.

The left translation of the same group gives a Poisson action on $\mathscr{M}$. The conservation of spatial moment $\boldsymbol{m}$ defines a level set $\mathscr{M}_{\boldsymbol{m}}$ of the momentum map $\boldsymbol{J}: \mathscr{M} \mapsto \mathfrak{g}^{*}$. The manifold $\mathscr{M}_{\boldsymbol{m}}$ is diffeomorphic to the group $\mathrm{SO}(3)$ itself and is right-invariant cross-sections of the cotangent bundle. The stationary subgroup $\mathscr{S}^{1} \in \mathrm{SO}(3)$, the circle, consists of rotations with respect to the direction $\mu$ of the vector $\boldsymbol{m}$. The actions of elements different from the identity of $\mathscr{S}^{1}$ 
on $\mathscr{M}_{\boldsymbol{m}}$ have no fixed point and leave this level set invariant. The reduced phase space is obtained from $\mathscr{M}_{m}$ by factoring by the action of the group $\mathscr{S}^{1}$, and can be identified with the momentum sphere $\mathscr{S}^{2}$ in $\mathbb{R}^{3}$ of Euclidean radius $\|\boldsymbol{m}\|$ as defined in Eq. (20). Clearly, this sphere is the base space of the unit circle bundle

$\pi_{m}: \mathscr{M}_{m} \mapsto \mathscr{M}_{m} / \mathscr{S}^{1} \simeq \mathscr{S}^{2}$,

with each fiber diffeomorphic to the group $\mathscr{S}^{1}$. This reduction process provides a simple frame work to reveal the geometry of the original fields $(\boldsymbol{\Lambda}(s), \boldsymbol{M}(s))$ lying on the level set $\mathscr{M}_{\boldsymbol{m}}$.

The material moment fields $\boldsymbol{M}(s)$ obtained by intersecting the energy ellipsoids with the angular momentum sphere give closed loops on the reduced space $\mathscr{S}^{2}$. After the field $\boldsymbol{M}(s)$ completes one period $S_{\sigma}$ in the base space, the original field $\left(\boldsymbol{\Lambda}\left(S_{\sigma}\right), \boldsymbol{M}\left(S_{\sigma}\right)\right)$ fails to return to its initial value $(\boldsymbol{\Lambda}(0), \boldsymbol{M}(0))$. However, since they are sitting on the same fiber $\pi_{m}^{-1}(\boldsymbol{M}(0))$, their relation can be determined by the action of the total phase $\alpha^{*} \in \mathscr{S}^{1}$, as has been expressed by Eq. (27)

$$
\boldsymbol{\Lambda}\left(S_{\sigma}\right)=\exp \left(\alpha^{*} \boldsymbol{\mu}\right) \cdot \boldsymbol{\Lambda}(0) .
$$

The geometric structure of the principal $\mathscr{S}^{1}$-bundle (Eq. (41)) allows us to decompose the total phase $\alpha^{*}$ into its geometric and dynamic part as expressed in Eq. (40). The canonical one form $\theta$ of the cotangent bundle $\mathscr{M}$ induces a natural connection one-form $\gamma=\frac{1}{\|\boldsymbol{m}\|} \theta$ on the principal bundle

$\pi_{m}: \mathscr{M}_{m} \mapsto \mathscr{S}^{2}$.

With this connection, there can be introduced an unique parallel translation along the path $\boldsymbol{M}(s)$ in $\mathscr{S}^{2}$

$$
\begin{aligned}
& p: s \mapsto\left(\boldsymbol{\Lambda}_{p}, \boldsymbol{M}\right) \in \mathscr{M}_{\boldsymbol{m}}, \\
& p(0) \simeq\left(\boldsymbol{\Lambda}_{p}(0), \boldsymbol{M}(0)\right)=(\boldsymbol{\Lambda}(0), \boldsymbol{M}(0)),
\end{aligned}
$$

such that the tangent vector $\dot{p}(s)$ to $p(s)$ is horizontal, i.e., $\gamma(\dot{p}(s))=0$, and that, for every $s$, this parallel translation $p(s)$ shares the same fiber with the real physical field

$\pi_{\boldsymbol{m}}(p(s))=\pi_{\boldsymbol{m}}(\boldsymbol{\Lambda}(s), \boldsymbol{M}(s))=\boldsymbol{M}(s)$.

Since $\boldsymbol{M}(s)$ is a closed curve in $\mathscr{S}^{2}$, by parallel translating all the element at the fiber $\pi_{\boldsymbol{m}}^{-1}(\boldsymbol{M}(0))$ along $\boldsymbol{M}(s)$, we obtain a map from $\pi_{m}^{-1}(\boldsymbol{M}(0))$ onto itself. From the uniqueness and smooth dependence on initial conditions, it follows that this map is a diffeomorphism, which is called the holonomy of the path $\boldsymbol{M}(s)$. The difference between $p\left(S_{\sigma}\right)$ and $p(0)$ gives rise to the geometric phase $\alpha_{g}$ appearing in the second term of Eq. (40). This group element $\alpha_{g} \in \mathscr{S}^{1}$ defines the holonomy along the path $\boldsymbol{M}(s)$, and can be computed from the well-known result

$\alpha_{g}=$ the holonomy of the connection $\gamma=-A$,

where $A$ is the solid angle subtended by the curve $\boldsymbol{M}(s)$.

For the dynamic phase that appeared in the first term of Eq. (40), we observe that, at each point $s$, the parallel translated curve $p(s)$ and the original fields $(\boldsymbol{\Lambda}(s), \boldsymbol{M}(s))$ lie on the same fiber; therefore, there is some curve in the structure group $s \mapsto \alpha_{d}(s) \in \mathscr{S}^{1}$ such that the original field can be reconstructed from its action on the parallel translation $p(s)$

$(\boldsymbol{\Lambda}(s), \boldsymbol{M}(s))=\exp \left(\alpha_{d}(s) \boldsymbol{\mu}\right) \cdot p(s)$.

The dynamic phase results from the difference between the true trajectory and the parallel translation at $S_{\sigma}$

$\alpha_{d}\left(S_{\sigma}\right)=\frac{2 W S_{\sigma}}{\|\boldsymbol{m}\|}$.

A direct derivation of Eqs. (42) and (43) based on the essential knowledge of connections and holonomy on fiber bundles has been provided by Marsden et al. [8]. However, as has been shown by Montgomery [12], the phase formula (Eq. (40)) can also be proved in an elementary manner by making use of the Stokes theorem. This proof will be discussed from a more geometric perspective in Appendix.

In view of the Kirchhoff kinetic analogy, the inherent geometry of the phase formula (Eq. (40)) is essentially the same as phases for the rigid body dynamics [8]. However, their physical content are quite different. The holonomy phenomena manifested in the spatial shapes of deformation seems to be more transparent than it in the time evolutions of a rigid body.

\section{Observable consequences}

In this section, several concrete examples are selected in order to illustrate the physical observable consequences of geometric phases. The first part are concerning transversely symmetric rods. By transversely symmetric it is meant that the constitutive relations (13) for the moment are symmetric to rotations about the axis $\boldsymbol{d}_{3}$. We will find that simple solutions can be derived for this particular case, and their corresponding mechanical phenomena are easier to understand. In this nice example, the phase formula (40) reduces to the familiar phase shift phenomenon occurring in a system consisting of two independent harmonic oscillators. In the second part, we will deal with the general case. Instead of deriving analytic solutions, the energy-momentums conserving numerical algorithms, which reflect the fundamental 
symmetry underlying these models, is adopted. We will observe that rules revealed in Sect. 3 are also implicit in those complex spatially equilibrium states resulting from numerical simulations.

\subsection{Theoretical analysis of symmetric cases}

Let us focus our attentions upon the case where $I_{1}=I_{2}=I$ and let $c=G J / E I$. The rods have identical bending stiffness in every direction, and the equation governing equilibrium states formally is equivalent to the free motion of spinning top. Abstract descriptions discussed in the previous part have simple explicit expressions in this case, which can serve as illustrations for our general theoretical study. To facilitate our discussion, the $z$-axis of the inertial frame $\boldsymbol{e}_{z}$ is taken in the direction of the constant angular momentum $\boldsymbol{m}$, i.e., $\boldsymbol{e}_{z}=\boldsymbol{m} / M$. We assume that the rod has circular cross-section, and in this particular case the Poisson's ratio $v$ equals to $\frac{1}{c}-1$. Following normal convention, let $v$ vary in the range $\left(0, \frac{1}{2}\right)$.

First of all, we note that, from the constitutive relations $M_{1}=E I \Omega_{1}$ and $M_{2}=E I \Omega_{2}$, the spatial rotational strain $\omega$ can be written as

$\omega=\frac{\boldsymbol{m}}{E I}+\Omega_{3}(1-c) \boldsymbol{t}$,

where $t:=d_{3}$ is the tangent of the center-curve, the component $\Omega_{3}$ is the twist or torsion angle. With help of this expression, we can write the derivative of $t$ along the centerline in the form

$\boldsymbol{t}^{\prime}=\omega \times \boldsymbol{t}=\frac{M}{E I}\left(\boldsymbol{e}_{z} \times \boldsymbol{t}\right)$

Two important facts concerning the behavior of $t$ can be drawn immediately. First, the $z$-component $t_{z}$ is a constant, for $t_{z}^{\prime}=\boldsymbol{e}_{z} \cdot \boldsymbol{t}^{\prime}=0$. This condition also results in constant torsion angle, $\Omega_{3}=$ constant. Second, the complex expression $t_{x}+\mathrm{i} t_{y}$ satisfies equation

$\left(t_{x}+\mathrm{i} t_{y}\right)^{\prime}=\frac{2 \pi \mathrm{i}}{S_{h}}\left(t_{x}+\mathrm{i} t_{y}\right)$,

where $S_{h}=2 \pi E I / M$. So $t_{x}+\mathrm{i} t_{y}=A \exp \left(2 \pi \mathrm{i} s / S_{h}\right)$, where $A$ is a complex constant depending on the value of $t$ at the clamped end.

These equations tells us that the tangent $t$ rotates uniformly about the direction of $\boldsymbol{m}$ with the rate $\Omega_{h}$, and the line itself is a twisted helix. Without loss of generality, we may assume the vector $t(0)$ at the clamped end takes the form

$\boldsymbol{t}(0)=\left[\begin{array}{lll}\sin \theta, & 0, \cos \theta\end{array}\right]$, where $\theta$ is the inclined angle of $\boldsymbol{t}$ with $\boldsymbol{e}_{z}$ direction. Therefore, the vector $t(s)$ describes a right circular cone of constant angle $\theta$ in space

$\boldsymbol{t}=\left[\sin (\theta) \cos \left(\frac{2 \pi s}{S_{h}}\right), \sin (\theta) \sin \left(\frac{2 \pi s}{S_{h}}\right), \cos (\theta)\right]$.

The parametrization of the center-line can be gotten by taking integration

$\boldsymbol{\varphi}(s)=\left[\rho \sin \left(\frac{2 \pi s}{S_{h}}\right), \rho\left(1-\cos \left(\frac{2 \pi s}{S_{h}}\right)\right), \zeta \frac{2 \pi s}{S_{h}}\right]$,

which is a helix, parameterized by the arc-length $s$, turning around a cylinder of radius $\varrho=S_{h} \sin (\theta) /(2 \pi)$, whose central axis points to the $z$-axis. The loop-to-loop distance of the helix is $\zeta=S_{h} \cos (\theta) /(2 \pi)$, and the length of the curve per turn is $S_{h}$.

Next, we need to calculate the distribution of stress $\boldsymbol{M}$. To do so, we expand the Euler Eq. (21) into component forms

$$
\begin{aligned}
M_{1}^{\prime}+\frac{G J-E I_{2}}{G J \cdot E I_{2}} M_{3} M_{2} & =0, \\
M_{2}^{\prime}+\frac{E I_{1}-G J}{G J \cdot E I_{1}} M_{3} M_{1} & =0, \\
M_{3}^{\prime} & =0 .
\end{aligned}
$$

In the symmetric case, we find the moment of twist $M_{3}=$ $\boldsymbol{m} \cdot \boldsymbol{d}_{3}=M \cos \theta$ is a constant. We can write the first two equations as

$\left(M_{1}+\mathrm{i} M_{2}\right)^{\prime}=-\frac{2 \pi \mathrm{i}}{S_{\sigma}}\left(M_{1}+\mathrm{i} M_{2}\right)$,

where $S_{\sigma}=2 \pi /\left(\Omega_{3}(1-c)\right)=S_{h} /(v \cos \theta)$. This result shows that the bending moment $\boldsymbol{M}_{b}=M_{1} \boldsymbol{d}_{1}+M_{2} \boldsymbol{d}_{2}$, rotates about the axis $\boldsymbol{d}_{3}$, remaining a constant magnitude. Since the torsion moment $M_{3}$ keeps constant, we conclude that the vector $\boldsymbol{M}$ rotates uniformly with period $S_{\sigma}$.

The dynamic and geometric phase could take the explicit forms in this particular case (noticing the sign of this signed solid angle):

$$
\begin{aligned}
\frac{2 W S_{\sigma}}{M} & =\frac{2 \pi S_{\sigma}}{S_{h}}\left(1+v \cos ^{2} \theta\right), \\
A & =-2 \pi(1-\cos \theta) .
\end{aligned}
$$

There are two distinguished periodic patterns: one is evidently the helical shape of the center line; the other concerns the periodic variation of $\boldsymbol{M}$. Each of them yields a phase function, one for $\varphi_{1}(s)=2 \pi s / S_{h}$, the other for $\varphi_{2}(s)=2 \pi s / S_{\sigma}$. 


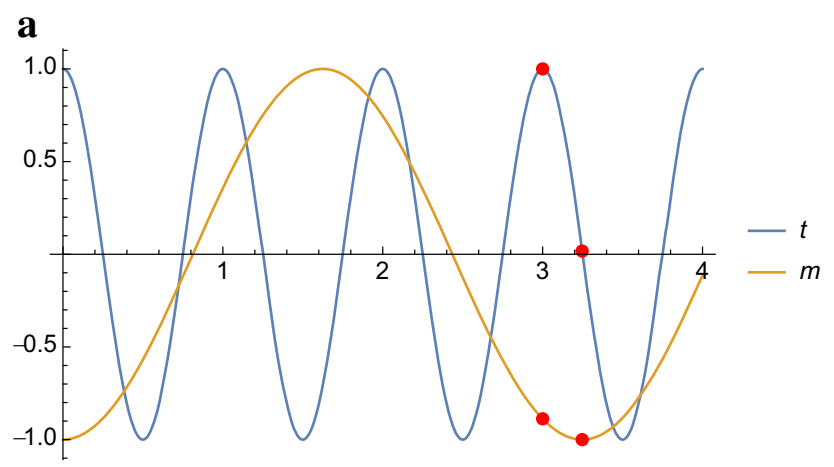

b

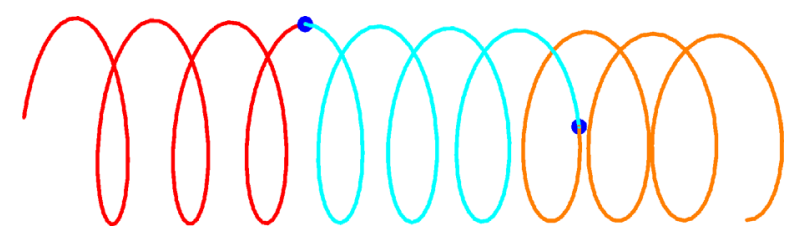

Helical center-line

Fig. 1 (Color online) Periodic pattern in symmetric case: two periodic patterns appearing in this case. One of them is the obviously helical shape. The other is periodic variation of $\boldsymbol{M}$. a The phase shift $2 \pi S_{\sigma} / S_{h}$ is, modulo $2 \pi$, the $\alpha^{*}$ defined in phase formula. b Blue points signify the separator of each segment divided according to the period of stress

The phase shift $\varphi_{1}\left(S_{\sigma}\right)$ during one period of $\varphi_{2}$ may be viewed as a manifestation of the phase formula (40), and modulo $2 \pi$, we have

$2 \pi \frac{S_{\sigma}}{S_{h}}=\frac{2 \pi}{v \cos \theta}=\left(\frac{2 W S_{\sigma}}{M}-A\right) \bmod 2 \pi$.

In Fig. 1a, two oscillations are presented to illustrate this phase shift behavior. One of them represents the distribution of $x$-component of tangent $t$, the other for the component $M_{1}$. Since we are just interested in the comparison of phase, unit amplitude are assumed for both cases.

When a point travels along the center-line, a length of $S_{\sigma}$, it will find the same situation repeated except the cross-section at this position has turned through an angle $2 \pi /(\nu \cos \theta)$ around the $\boldsymbol{m}$ axis. A second movement will be exactly like the first (see Fig. 1b).

\subsection{Numerical simulations of general cases}

To appreciate the physical implications of our theoretical results in general cases, let us now proceed to the numerical solutions of the equilibriums. The problem mentioned at the beginning of Sect. 3 is apparently a boundary value problem. At first glance, it seems necessary to use finite element methods to obtain the equilibrium solutions. However, spatial deformation patterns concerned with here are exhibited by infinitely or arbitrary long rod. With the increase of the rod's length, the dimension of tangent stiffness matrices constructed from sufficiently refined mesh tend to be considerably large, and the cost to invert them become expensive. On the another hand, if the initial guess is not sufficiently close to the solution, typical iterative solvers for nonlinear equations could fail to converge. Since the deformed shapes under our study are very complex, a reasonable initial guess cannot be easily obtained. Therefore, solving this problem by finite element methods is clearly not the wise approach.

Fortunately, a Kirchhoff kinetic analogy leads to a welldefined initial value problem. To solve this problem, the only relevant equations are

$\boldsymbol{\Lambda}^{\prime}=\boldsymbol{\Lambda} \boldsymbol{\Omega}$ and $\mathbb{J} \boldsymbol{\Omega}^{\prime}=\mathbb{J} \boldsymbol{\Omega} \times \boldsymbol{\Omega}$,

which is the classical Euler's equation describing the rotation dynamics. To get a neat expression, the symbol $\mathbb{J}$ is introduced to represent the elastic modulus $\boldsymbol{C}_{\boldsymbol{M}}$. The initial value of $\boldsymbol{\Lambda}$ is obviously imposed by the boundary value at the clamped end (see Eq. (14)). The invariance of the moment vector $\boldsymbol{m}$ immediately yields the initial value of $\boldsymbol{\Omega}$

$\boldsymbol{\Omega}(0)=\mathbb{J}^{-1} \boldsymbol{\Lambda}^{\mathrm{T}}(0) \boldsymbol{m}$.

To sum up, the ordinary differential equations (52) together with the initial data

$\boldsymbol{\Lambda}(0)=\boldsymbol{\Lambda}_{0} \quad$ and $\quad \boldsymbol{\Omega}(0)=\mathbb{J}^{-1} \boldsymbol{\Lambda}_{0}^{\mathrm{T}} \boldsymbol{m}$

comprise a initial value problem.

The numerical method adopted here is the single time stepping algorithms, advocated by Simo and Wong [19], for the orthogonal group which conserves energy and momentum. More specifically, we regard an algorithm as a map $F_{h}: \mathrm{SO}(3) \times \mathbb{R}^{3} \mapsto \mathrm{SO}(3) \times \mathbb{R}^{3}$, which depends smoothly on the time step $h$, such that

$\left(\boldsymbol{\Lambda}_{n+1}, \boldsymbol{\Omega}_{n+1}\right)=F_{h}\left(\boldsymbol{\Lambda}_{n}, \boldsymbol{\Omega}_{n}\right)$.

The energy and momentum vector are also regarded as functions defined on the manifold $\mathrm{SO}(3) \times \mathbb{R}^{3}$ :

$W(\boldsymbol{\Lambda}, \boldsymbol{\Omega})=\frac{1}{2} \boldsymbol{\Omega} \cdot \mathbb{\Omega} \boldsymbol{\Omega}$ and $\boldsymbol{m}(\boldsymbol{\Lambda}, \boldsymbol{\Omega})=\boldsymbol{\Lambda} \cdot \mathbb{J} \boldsymbol{\Omega}$.

An algorithm is said to conserve the energy and momentum, if $W \circ F_{h}=W$ and $\boldsymbol{m} \circ F_{h}=\boldsymbol{m}$. This condition tells us that the following relations are guaranteed to hold at each step $s_{n}$

$$
\begin{gathered}
W\left(\boldsymbol{\Lambda}_{n+1}, \boldsymbol{\Omega}_{n+1}\right)=W\left(\boldsymbol{\Lambda}_{n}, \boldsymbol{\Omega}_{n}\right), \\
\boldsymbol{m}\left(\boldsymbol{\Lambda}_{n+1}, \boldsymbol{\Omega}_{n+1}\right)=\boldsymbol{m}\left(\boldsymbol{\Lambda}_{n}, \boldsymbol{\Omega}_{n}\right) .
\end{gathered}
$$


Accordingly, the discrete sequence of material moment (angular momentum in the body) $\left\{\boldsymbol{M}_{n}=\mathbb{J} \boldsymbol{\Omega}_{n}\right\}_{n=1,2 \ldots}$ lies on the intersection of the sphere with radius $M=\|\boldsymbol{m}\|$ and the surface of constant energy. This algorithm is singularityfree and integrates the dynamics exactly up to a time reparametrization. The detailed constructions are referred to in Ref. [19].

With discrete solutions $\left\{\boldsymbol{\Lambda}_{n}\right\}_{n=1,2 \ldots}$ in hand, the geometry of the center line could be constructed through direct numerical quadrature of the rod's axis $t$

$\boldsymbol{\varphi}\left(s_{n}\right)=\int_{0}^{s_{n}} \boldsymbol{t} \mathrm{d} s=\sum_{k=1}^{n-1} \frac{1}{2}\left(\boldsymbol{t}_{k+1}+\boldsymbol{t}_{k}\right)\left(s_{k+1}-s_{k}\right)$,

where the vector $\boldsymbol{t}_{k}$ can be identified with the third column of the matrix $\boldsymbol{\Lambda}$, i.e., $\boldsymbol{t}_{k}=\boldsymbol{\Lambda}_{k} \boldsymbol{e}_{3}$.

Let us proceed to use the energy-momentum conserving algorithm to illustrate deformation patterns of the rod under the type of boundary condition specified by the Eqs. (14) and (15). The numerical constants used in the simulations are given as follows

$\boldsymbol{m}=(0,0,2 \pi), \quad\left(E I_{1}, E I_{2}, G J\right)=(1, r, 2)$,

where the parameter $r$ represents the ratio $E I_{2} / E I_{1}$. The initial conditions are specified by the inclined angle $\theta_{0}$, i.e., the angle between the initial tangent $t_{0}$ and the direction of external moment $\boldsymbol{m}$

$\boldsymbol{\Lambda}_{0}=\left[\begin{array}{ccc}\cos \left(\theta_{0}\right) & 0 & \sin \left(\theta_{0}\right) \\ 0 & 1 & 0 \\ -\sin \left(\theta_{0}\right) & 0 & \cos \left(\theta_{0}\right)\end{array}\right], \quad \boldsymbol{\Omega}_{0}=\left[\begin{array}{c}-2 \pi \sin \left(\theta_{0}\right) \\ 0 \\ \pi \cos \left(\theta_{0}\right)\end{array}\right]$.

The energy constant, in view of Eq. (22), can be written in terms of $\theta_{0}$

$W\left(\theta_{0}\right)=\pi^{2}\left(1+\sin ^{2} \theta_{0}\right)$,

and the semi-axes of the energy ellipsoid (24) are respectively $\sqrt{2 W\left(\theta_{0}\right)}, \sqrt{2 r W\left(\theta_{0}\right)}$, and $\sqrt{4 W\left(\theta_{0}\right)}$. The length of rod $L$ is insignificant here, it just needs to be large enough to contain several periods for each solution.

Let us fix the initial inclined angle $\theta_{0}$ to be $\pi / 6$, and examine the way in which the deformed state of the rod changes as $r$ varies. For $r=1$, the simulation recovers the symmetric case and produces a helical shape as discussed in the previous part, (see Eq. (48)). For other values of $r$, a sample of results is presented below. In all of these simulations, the time-step size is $h=0.01$.

First, several orbits of the material moment $\boldsymbol{M}$ are exhibited in Fig. 2. A distinguished value $r_{\star}:=M^{2} /(2 W)$ of the parameter $r$ should be noticed here. For the case $\theta_{0}=\pi / 6$ considered here, $r_{\star}=1$.6. When $r$ is smaller then $r_{\star}$, paths of

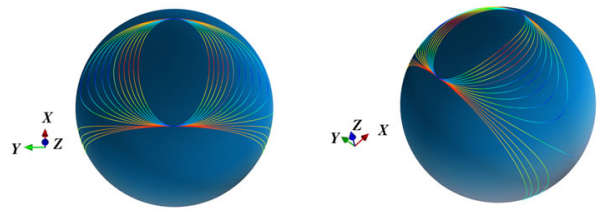

Fig. 2 The orbit of moment referred to the body frame

$\mathbf{a}$

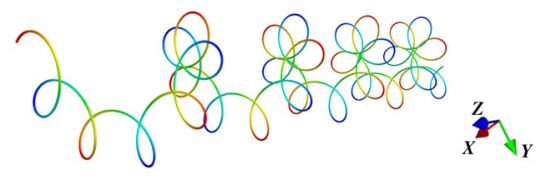

b

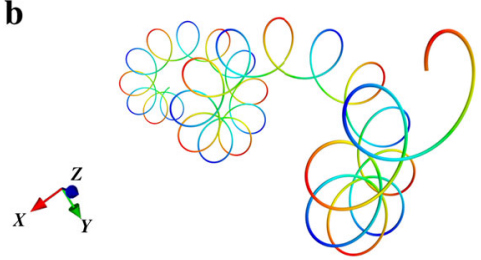

c

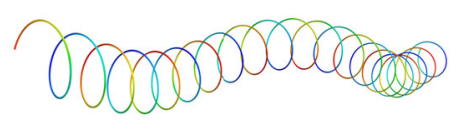

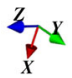

d

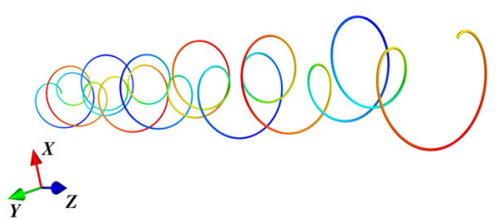

Fig. 3 Typical shapes of center-lines. $\mathbf{a} r=0.5$. $\mathbf{b} r=0.6$. $\mathbf{c} r=0.9$. d $r=1.4$

the terminus of $\boldsymbol{M}$ is a closed curve round the $\boldsymbol{d}_{3}$ axis. When $r$ increases, the curve become larger, and when it reaches the point $r_{\star}$, the intersection of the sphere of constant angular momentum magnitude $M$ with the elliptical energy surface consists of two great circles on the sphere going through the $M_{2}$-axis. However, the vector $\boldsymbol{M}$ moves from the initial position $\boldsymbol{M}_{0}$ asymptotically to the $M_{2}$-axis. The orbit is a part of the great circle, and the variation of $\boldsymbol{M}$ is not periodic. When $r$ increases further, closed paths again appear, but now around the $-\boldsymbol{d}_{1}$ axis.

In Fig. 3, several typical deformed shapes are listed. A clear idea of the nature of geometric phases can be extracted by investigating the geometry of these configurations. Let us consider the change of the tangent $t$ of the center-line during one stress period $S_{\sigma}$. To study the orbit of $t$ in space, we use the Euler angles $\phi_{1}, \theta, \psi$ between the body frame $\boldsymbol{d}_{1}, \boldsymbol{d}_{2}, \boldsymbol{d}_{3}$, and the axes $\boldsymbol{e}_{x}, \boldsymbol{e}_{y}, \boldsymbol{e}_{z}$ of the fixed system of coordinates, taking the $\boldsymbol{e}_{z}$-axis in the direction of the constant vector $\boldsymbol{m}$. The conventions adopted here follow the ones used by Landau and Lifshitz [20] and Arnol'd [16]. 
Since the polar angle and azimuth of the axis $\boldsymbol{e}_{z}$ with respect to $\boldsymbol{d}_{1}, \boldsymbol{d}_{2}, \boldsymbol{d}_{3}$ are respectively, $\theta$ and $\frac{\pi}{2}-\psi$, the components of $\boldsymbol{M}$, i.e., the components of $\boldsymbol{m}$ along the axes $\boldsymbol{d}_{1}, \boldsymbol{d}_{2}, \boldsymbol{d}_{3}$, can be expressed in terms of $\theta$ and $\psi$

$M_{1}=M \sin \theta \sin \psi, M_{2}=M \sin \theta \cos \psi, M_{3}=M \cos \theta$.

Hence,

$\cos \theta=\frac{M_{3}}{M}, \quad \tan \psi=\frac{M_{1}}{M_{2}}$.

The periodicity of $\boldsymbol{M}$ immediately implies the angles $\theta$ and $\psi$ are periodic functions with stress-period $S_{\sigma}$.

The position of the tangent vector $\boldsymbol{t}$ is determined by its polar angle and azimuth referring to the fixed coordinates axes $\boldsymbol{e}_{x}, \boldsymbol{e}_{y}, \boldsymbol{e}_{z}$. They are respectively $\theta$ and $\phi:=\phi_{1}-\frac{\pi}{2}$. As mentioned above, the inclination $\theta$ of the $\boldsymbol{t}$ to the vertical is a periodic function with period $S_{\sigma}$. However, the variation of azimuth $\phi$ of the tangent does not follow the similar periodic pattern, and after a period $S_{\sigma}$, it varies by an angle given by the phase formula (40)

$\phi\left(S_{\sigma}\right)=\left(\frac{2 W S_{\sigma}}{M}-A\right) \bmod 2 \pi$.

The change of $\theta$ and $\phi$ during one period is of the kind shown in Fig. 4.

The terminus of $\boldsymbol{t}$ moves in the ring between the parallels $\theta_{\min }$ and $\theta_{\max }$. The condition for its path to be closed is that the angle $\phi\left(S_{\sigma}\right)$ should be a fractional of $2 \pi$, i.e., $\phi\left(S_{\sigma}\right)=$ $2 \pi m / n$. In that case, after $n$ periods, the vector $t$ will occupy its original position, so that the path is closed. However, such cases are exceptional. In general, therefore, the path of $t$ is not closed, and it repeatedly passed through the $\theta_{\min }$ and $\theta_{\max }$. After many times, $\boldsymbol{t}$ will covers the entire band between the two bounding circle without exactly returning to its original position. In Fig. 5, several examples are presented to illustrate orbits of the tangent vector $\boldsymbol{t}$.

As we have discussed concisely in Sect. 3, a better physical understanding of the phase formula (Eq. (40)) can be obtained
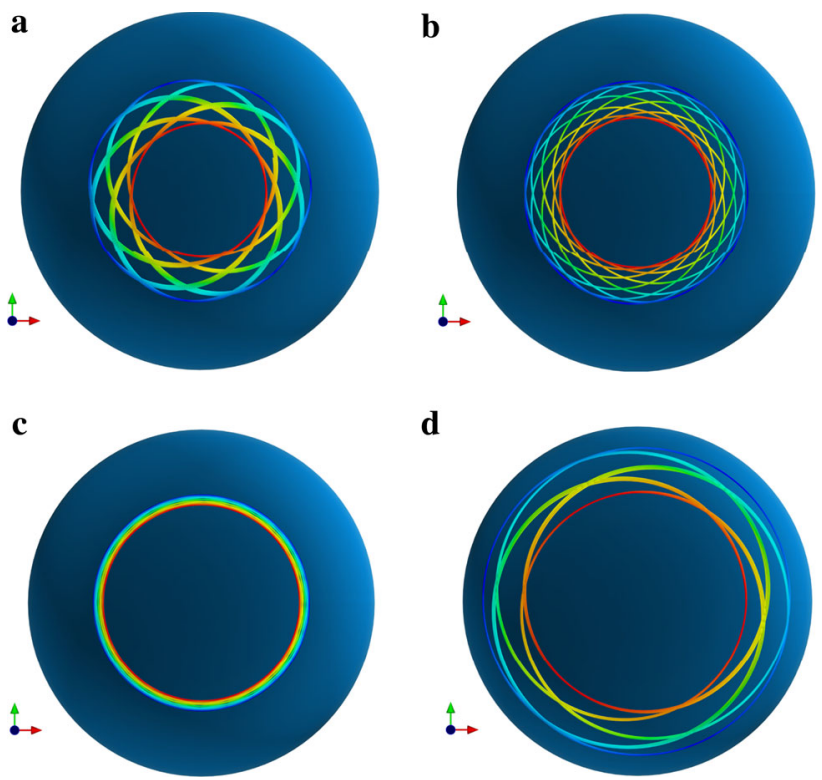

Fig. 5 Paths of the tangent $t$ in the unit sphere. $\mathbf{a} r=0.5$. $\mathbf{b} r=0.6$. c $r=0.9$. d $r=1.4$

from the study of current configurations of the underlying rod model. We presented the behavior of the case $r=0.5$ in a visual way to illustrate this abstract idea.

The angle $\phi\left(S_{\sigma}\right)$ and the position vector $\varphi\left(S_{\sigma}\right)$ together define a rigid-body motion via

$(\boldsymbol{R}, \boldsymbol{u})=\left(\exp \left(\phi\left(S_{\sigma}\right) \boldsymbol{e}_{z}\right), \boldsymbol{\varphi}\left(S_{\sigma}\right)\right)$

Figure 6a shows the projection onto the $x y$-plane of a part of the deformed center line from 0 to $S_{\sigma}$, defined via the restriction of the map $\varphi$

$\varphi_{[1]}=\left.\varphi\right|_{\left[0, S_{\sigma}\right]}$.

As we claimed in Eq. (36), the segment $\varphi_{[2]}$ can be obtained directly from $\varphi_{[1]}$ through the affine transformation $(\boldsymbol{R}, \boldsymbol{u})$

$\left(\varphi_{[2]}, \Lambda_{[2]}\right)=\left(\boldsymbol{R} \varphi_{[1]}+\boldsymbol{u}, \boldsymbol{R} \Lambda_{[1]}\right)$

b

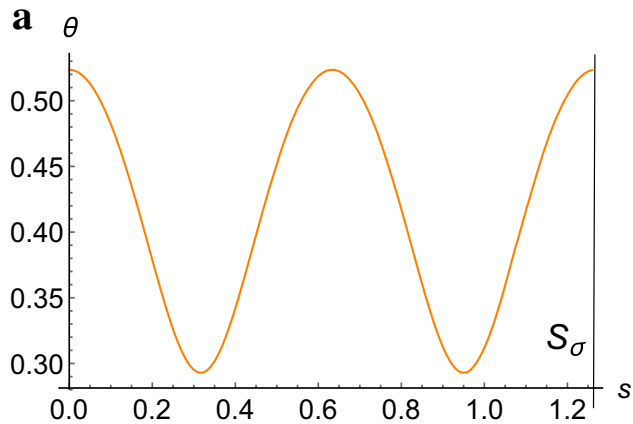

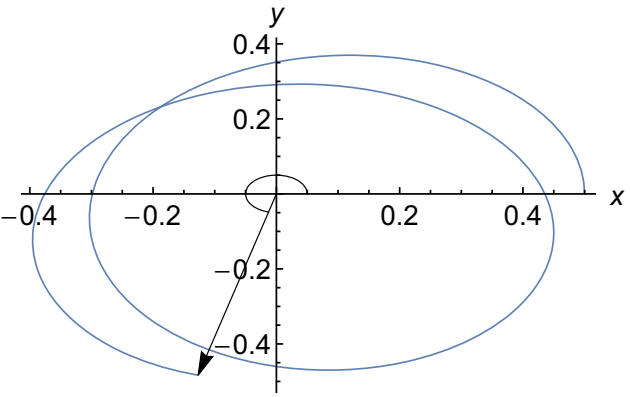

Fig. 4 The variation of $\theta$ and $\phi$ during one stress-period $S_{\sigma}$. a The change of $\theta$. b The change of $\phi$ 

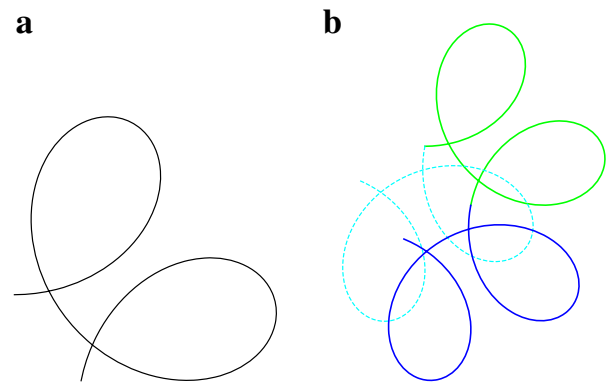

Fig. 6 (Color online) The process of affine transformation that produces $\varphi_{[2]}$ from $\varphi_{[1]}$. In plot $\mathbf{b}$, the green line is the $x y$-projection of $\boldsymbol{\varphi}_{[1]}$, the dashed line is the rotation of $\boldsymbol{\varphi}_{[1]}$ around the $\boldsymbol{e}_{z}$ axis through an angle $\phi\left(S_{\sigma}\right)$, and the blue line gives the visual representation of $\boldsymbol{\varphi}_{[2]}$. a $\varphi_{[1]} \cdot \mathbf{b} \varphi_{[1]} \mapsto \varphi_{[2]}$ a

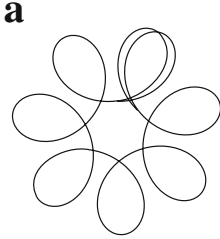

b

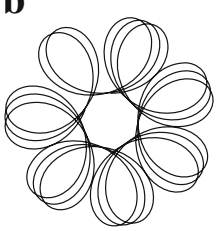

c

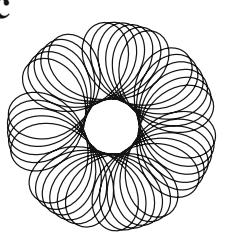

Fig. 7 The $x y$-plane projection of the center-line with $r=0.5$ for several period. a 4 period. b 10 period. c 20 period
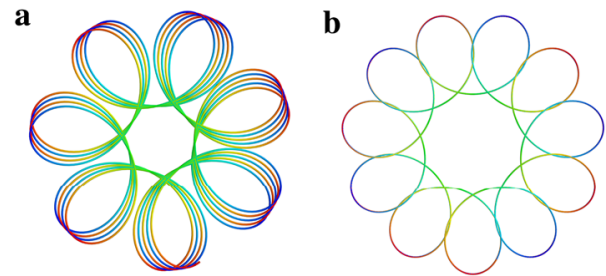

c

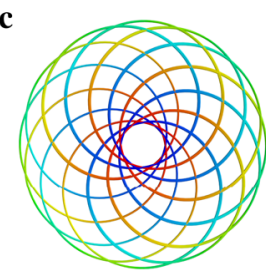

d

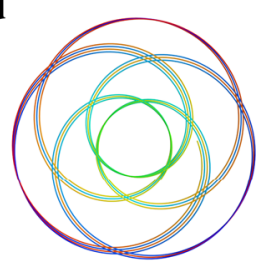

Fig. 8 The $x y$-plane projections of center-lines for typical $r$ values. $\mathbf{a} r=0.5$. $\mathbf{b} r=0.6$. $\mathbf{c} r=0.9$. $\mathbf{d} r=1.4$

Figure $6 \mathrm{~b}$ is used to illustrate this process.

Figure 7 shows what happens when one starts with just one segment $\varphi_{[1]}$ and then applies the affine transformation $(\boldsymbol{R}, \boldsymbol{u})$ over and over again. What one see is quite startling. The complex deformed shape of the center-line is build gradually from the simple rule specified in Eq. (38).

Figure 8 presents several $x y$-plane projections of centerlines with different $r$ values. Despite their apparent intricate behavior, the underlying rule for them is simply given in Eq. (38) similar to the case $r=0.5$ discussed before.

\section{Conclusions}

In this paper, we have analyzed, within the framework of geometrically exact rod theory, the deformed shapes of equilibrium states subjected to external moment of the forces applied at the free end. It follows from the Kirchhoff kinetic analogy that the material moment, and hence, the strain of the rod is periodic along the length of the rod. When the material moment vector returns to its original state, the configuration acquires a rotation with a nontrivial angle, which records not only informations of the local deformation experienced by the rod, i.e., dynamics phase, but also the peculiar structure associated with the global geometric nature of the closed curve traced by the material moment vector. The significance of this results allow us to break the rod down to find its underlying segments. Then the whole deformed rod can be build up through affine transformation of each individual part. The numerical results in Sect. 4 confirm this insight shed by the geometric phases and illustrate how the complex patterns exhibited by the rod deformation could be understood with the help of our theoretic findings.

The helical and helix-like shapes are ubiquitous both in nature and in artificial objects, and can be found on many scales from microscopic structures such as $\alpha$-helices in protein, nanowires, and the famous DNA double helix to macroscopic structures such as human hairs, springs, and tendrils of plants. In particular, the geometric exact rod model provides a reasonable approximation of these soft and filamentary bodies. Therefore, it is very plausible to expect that the geometric phases here have some very significant roles to play in these structures from the physical world. The potential practical applications of geometrical phases in the fields such as nano-science and polymer science should deserve serious studies.

\section{Appendix}

From the view of mathematics, the exterior differential forms give unique insight into the geometry of the mechanical problem concerned with here. The concept of differential form, and the mathematical formalism for manipulating them, called exterior calculus, arise when concepts such as the work of a force along a path and the flux of a fluid through a surface are generalized to a curved manifold. Readers who have not been familiar with these mathematical tools should refer to the introductory materials presented in excellent books of Arnol'd [16] and Misner et al. [21].

The key to understanding this result is based upon the integration of the right invariant 1 -form $m^{b}$ associated with the conserved moment vector $\boldsymbol{m}$. Identifying the tangent bundle $T \mathrm{SO}$ (3) with the right trivialization $\mathrm{SO}(3) \times \mathbb{R}^{3}$ (physically, using spatial variable), the vector $\boldsymbol{\theta}_{\boldsymbol{\Lambda}} \in T_{\boldsymbol{\Lambda}} \mathrm{SO}$ (3) has repre- 


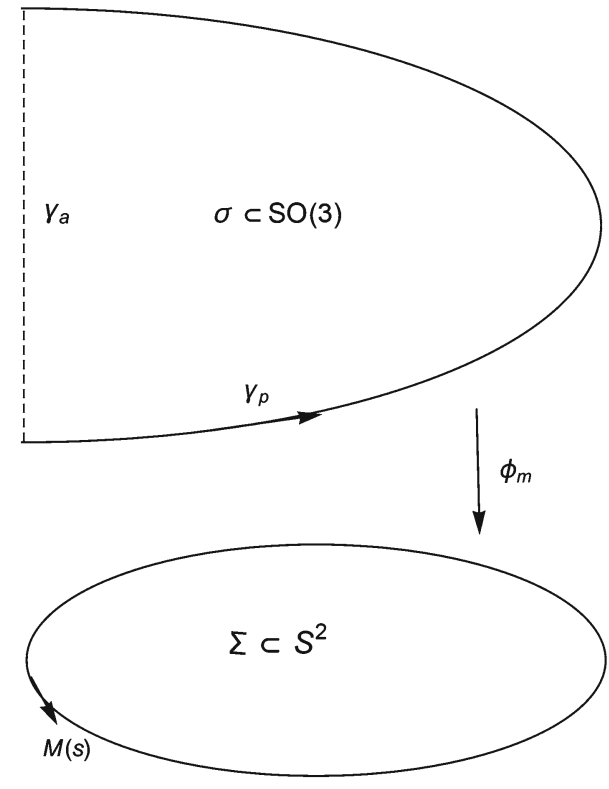

Fig. 9 Curves and surfaces in the proof

sentation $\hat{\boldsymbol{\theta}} \boldsymbol{\Lambda}$. Then the 1 -form $\left.m^{\mathrm{b}}\right|_{\boldsymbol{\Lambda}} \in T_{\boldsymbol{\Lambda}}^{*} \mathrm{SO}$ (3) at the point $\boldsymbol{\Lambda}$ is naturally defined via

$m^{b}\left(\boldsymbol{\theta}_{\boldsymbol{\Lambda}}\right)=\boldsymbol{m} \cdot \boldsymbol{\theta}$ for all $\boldsymbol{\theta}_{\boldsymbol{\Lambda}} \in T_{\boldsymbol{\Lambda}} \mathrm{SO}(3)$.

Consider the following two curves lying on $\mathrm{SO}(3)$. The first is the physical curve defined by the rotation field of the rod (Fig. 9)

$\gamma_{p}: s \mapsto \boldsymbol{\Lambda}(s)$ for $s \in\left[0, S_{\sigma}\right]$.

In order to constructed a closed curve, we define an auxiliary curve to connect the two end points of $\gamma_{p}$

$\gamma_{a}: s \mapsto \exp (\alpha \boldsymbol{\mu}) \boldsymbol{\Lambda}(0)$ for $\alpha \in\left[0, \alpha^{*}\right]$,

which satisfies $\gamma_{p}(0)=\gamma_{a}(0)$ and $\gamma_{p}\left(S_{\sigma}\right)=\gamma_{a}\left(\alpha^{*}\right)$. Therefore, $\gamma=\gamma_{p}-\gamma_{a}$ (the curve obtained by first going along $\gamma_{p}$ and then backward along $\gamma_{a}$ ) is a closed curve on the $\mathrm{SO}(3)$. The mystery hidden in Eq. (40) can be revealed through the investigation of the integral of the 1 -form $m^{b}$ along $\gamma$ :

$\int_{\gamma} m^{\mathrm{b}}=\int_{\gamma_{p}} m^{\mathrm{b}}-\int_{\gamma_{a}} m^{\mathrm{b}}$

First, we consider the integral $\int_{\gamma_{p}} m^{b}$ along the physical curve. The tangent of $\gamma_{p}$ can be expressed as $\boldsymbol{\omega} \boldsymbol{\Lambda}$. Noting that $m^{b}(\boldsymbol{\omega} \boldsymbol{\Lambda})=\boldsymbol{m} \cdot \boldsymbol{\omega}=2 W$, we obtain

$$
\int_{\gamma_{p}} m^{\mathrm{b}}=\int_{0}^{S_{\sigma}} m^{\mathrm{b}}(\boldsymbol{\omega} \boldsymbol{\Lambda}) \mathrm{d} s=2 W S_{\sigma} .
$$

Next, for the auxiliary curve $\gamma_{a}$, its tangent is simply the form $\boldsymbol{\mu} \exp (\alpha \boldsymbol{\mu})$. Since

$m^{b}(\boldsymbol{\mu} \exp (\alpha \boldsymbol{\mu}))=\boldsymbol{m} \cdot \boldsymbol{\mu}=M$

the value of the integral of $m^{\mathrm{b}}$ along it can be obtained

$\int_{\gamma_{a}} m^{b}=\int_{0}^{\alpha^{*}} m^{b}(\boldsymbol{\mu} \exp (\alpha \boldsymbol{\mu})) \mathrm{d} \alpha=M \alpha^{*}$.

Finally, with previous results, the integral around the closed curve $\gamma$ takes the form

$\int_{\gamma} m^{b}=2 W S_{\sigma}-M \alpha^{*}$

By applying the Stokes' theorem, we can relate the integral $\int_{\gamma} m^{b}$ to the integral over an arbitrary surface $\sigma \subset \operatorname{SO}(3)$ encircled by $\gamma$, i.e., $\partial \sigma=\gamma$

$\int_{\sigma} \mathrm{d} m^{b}=\int_{\gamma} m^{b}$

In order to obtain the relation Eq. (40), we just need to prove that

$\int_{\sigma} \mathrm{d} m^{b}=M A$

We establish the linkage between quantities on the left and right hand side of Eq. (A9). To do so, we first need to compute the 2 -form $\mathrm{d} m^{\mathrm{b}}$. This can be done by employing two arbitrary right invariant vector fields $\boldsymbol{\xi}_{\boldsymbol{\Lambda}}$ and $\boldsymbol{\eta}_{\boldsymbol{\Lambda}}$. Bearing in mind that $\left[\boldsymbol{\xi}_{\boldsymbol{\Lambda}}, \boldsymbol{\eta}_{\boldsymbol{\Lambda}}\right]=-\operatorname{skew}(\boldsymbol{\xi} \times \boldsymbol{\eta}) \boldsymbol{\Lambda}$, we obtain

$$
\begin{aligned}
\mathrm{d} m^{\mathrm{b}}\left(\boldsymbol{\xi}_{\boldsymbol{\Lambda}}, \boldsymbol{\eta}_{\boldsymbol{\Lambda}}\right) & =\boldsymbol{\xi}_{\boldsymbol{\Lambda}}\left(m^{\mathrm{b}}\left(\boldsymbol{\eta}_{\boldsymbol{\Lambda}}\right)\right)-\boldsymbol{\eta}_{\boldsymbol{\Lambda}}\left(m^{\mathrm{b}}\left(\boldsymbol{\xi}_{\boldsymbol{\Lambda}}\right)\right)-m^{\mathrm{b}}\left(\left[\boldsymbol{\xi}_{\boldsymbol{\Lambda}}, \boldsymbol{\eta}_{\boldsymbol{\Lambda}}\right]\right) \\
& =-m^{\mathrm{b}}\left(\left[\boldsymbol{\xi}_{\boldsymbol{\Lambda}}, \boldsymbol{\eta}_{\boldsymbol{\Lambda}}\right]\right) \\
& =\boldsymbol{m} \cdot(\boldsymbol{\xi} \times \boldsymbol{\eta}) .
\end{aligned}
$$

Note that although this calculation is carried out with help of the right invariant vector field, values of $\mathrm{d} m^{b}$ just linearly depend on vectors at $T_{\boldsymbol{A}} \mathrm{SO}(3)$. Therefore, $\mathrm{d} m^{b}$ is determined via the relation

$\mathrm{d} m^{\mathrm{b}}\left(\boldsymbol{\xi}_{\boldsymbol{\Lambda}}, \boldsymbol{\eta}_{\boldsymbol{\Lambda}}\right)=\boldsymbol{m} \cdot(\boldsymbol{\xi} \times \boldsymbol{\eta})$

for all $\boldsymbol{\xi}_{\boldsymbol{\Lambda}}, \boldsymbol{\eta}_{\boldsymbol{\Lambda}} \in T_{\boldsymbol{\Lambda}} \mathrm{SO}(3)$.

With an appropriate choice of a surface $\sigma \subset \mathrm{SO}(3)$, the map $\left.\phi_{\boldsymbol{m}}\right|_{\sigma}$, which is the restriction of $\phi_{\boldsymbol{m}}$ on the surface $\sigma$, is an diffeomorphism to the surface $\Sigma \subset \mathscr{S}^{2}$ capping the reduced closed curve defined by image of the map $\phi_{\boldsymbol{m}} \circ \gamma$. Let $\psi$ denote the inverse $\left(\left.\phi_{m}\right|_{\sigma}\right)^{-1}: \Sigma \mapsto \sigma$, according to 
the change of variables theorem, we have

$$
\int_{\sigma} \mathrm{d} m^{b}=\int_{\Sigma} \psi^{*} \mathrm{~d} m^{b} .
$$

The integral on the surface $\Sigma$ can be calculated by taking the following strategy. We note that every differential 2-form on the sphere can be written in the form $f \varepsilon$, where $\varepsilon$ is the standard area element of $\mathscr{S}^{2}$, and $f$ is a scalar function. In particular, the pull back of $\mathrm{d} \mathrm{m}^{\mathrm{b}}$ can be written into this form

$\psi^{*} \mathrm{~d} m^{b}=K \varepsilon$

where $K$ is a smooth function on the subset $\Sigma$ of $\mathscr{S}^{2}$.

To determine the function $K$, let us first take an arbitrary point $z \in \Sigma$ and choose two unit vectors $\boldsymbol{x}, \boldsymbol{y} \in T_{z} \mathscr{S}^{2}$ tangent to $\mathscr{S}^{2}$ at the point $z$, which satisfy the relations

$\boldsymbol{x} \cdot \boldsymbol{y}=0$ and $\boldsymbol{x} \times \boldsymbol{y}=\boldsymbol{n}$,

where $\boldsymbol{n}$ is the normal to $\mathscr{S}^{2}$ at $\boldsymbol{z}$. Here two facts need to be keep in mind: the first is that the definition of standard volume form gives us $\varepsilon(\boldsymbol{x}, \boldsymbol{y})=1$; the second is that the matrix $\psi(z)$ rotate the normal $\boldsymbol{n}$ to the direction of $\boldsymbol{m}$, i.e., $\boldsymbol{\mu}=\psi(z) \boldsymbol{n}$.

Let us denote $\boldsymbol{\Lambda}$ to be the rotation matrix $\psi(\boldsymbol{z})$, and take the vectors $\xi_{\boldsymbol{\Lambda}}, \eta_{\boldsymbol{\Lambda}} \in T_{\boldsymbol{\Lambda}} \mathrm{SO}(3)$ which are the images of the vectors $\boldsymbol{x}, \boldsymbol{y}$ under the tangent map $T_{z} \psi$

$\boldsymbol{\xi}_{\boldsymbol{\Lambda}}=T_{z} \psi \cdot \boldsymbol{x}, \quad \eta_{\boldsymbol{\Lambda}}=T_{z} \psi \cdot \boldsymbol{y}$.

Since the value of $\psi^{*} \mathrm{~d} m^{b}(\boldsymbol{x}, \boldsymbol{y})$ is defined by the equation

$\psi^{*} \mathrm{~d} m^{b}(\boldsymbol{x}, \boldsymbol{y})=\mathrm{d} m^{b}\left(\boldsymbol{\xi}_{\boldsymbol{\Lambda}}, \boldsymbol{\eta}_{\boldsymbol{\Lambda}}\right)$,

and $\varepsilon(\boldsymbol{x}, \boldsymbol{y})=1$, the value $K$ at the point $z$ can be determined by the relation

$K(\boldsymbol{z})=\mathrm{d} m^{\mathrm{b}}\left(\boldsymbol{\xi}_{\boldsymbol{\Lambda}}, \boldsymbol{\eta}_{\boldsymbol{\Lambda}}\right)$

By recalling that $\phi_{\boldsymbol{m}}(\boldsymbol{\Lambda})=\boldsymbol{\Lambda}^{\mathrm{T}} \boldsymbol{m}$, for an arbitrary vector $\boldsymbol{\theta}_{\boldsymbol{\Lambda}} \in T_{\boldsymbol{\Lambda}} \mathrm{SO}(3)$, the transformation of $\boldsymbol{\theta}_{\boldsymbol{\Lambda}}$ under the tangent map of $\phi_{\boldsymbol{m}}$ at $\boldsymbol{\Lambda}$ takes a simple form

$T_{\boldsymbol{\Lambda}} \phi_{\boldsymbol{m}} \cdot \boldsymbol{\theta}_{\boldsymbol{\Lambda}}=\boldsymbol{\Lambda}^{\mathrm{T}}(\boldsymbol{m} \times \boldsymbol{\theta})$.

Therefore, we have

$\boldsymbol{m} \times \xi=\boldsymbol{\Lambda} \boldsymbol{x}$ and $\boldsymbol{m} \times \boldsymbol{\eta}=\boldsymbol{\Lambda} \boldsymbol{y}$.

By taking the cross product of $\boldsymbol{m} \times \boldsymbol{\xi}$ and $\boldsymbol{m} \times \boldsymbol{\eta}$, we find

$(\boldsymbol{m} \times \xi) \times(\boldsymbol{m} \times \eta)=\Lambda(x \times y)=\Lambda n=\mu$.
However, with the help of the identity of the cross product and Eq. (A10) concerning $\mathrm{d}^{\text {b }}$, the left hand side can also be expressed in the form

$(\boldsymbol{m} \times \boldsymbol{\xi}) \times(\boldsymbol{m} \times \boldsymbol{\eta})=(\boldsymbol{m} \cdot(\boldsymbol{\xi} \times \boldsymbol{\eta})) \boldsymbol{m}=\mathrm{d} m^{\mathrm{b}}\left(\boldsymbol{\xi}_{\boldsymbol{\Lambda}}, \boldsymbol{\eta}_{\boldsymbol{\Lambda}}\right) \boldsymbol{m}$

Comparison of $\boldsymbol{\mu}$ and $\mathrm{d} m^{\mathrm{b}}\left(\boldsymbol{\xi}_{\boldsymbol{\Lambda}}, \boldsymbol{\eta}_{\boldsymbol{\Lambda}}\right) \boldsymbol{m}$ leads to

$K(z)=\mathrm{d} m^{\mathrm{b}}\left(\boldsymbol{\xi}_{\boldsymbol{\Lambda}}, \boldsymbol{\eta}_{\boldsymbol{\Lambda}}\right)=1 / M$

Since the choice of $z$ is arbitrary, this result holds for all poinst $z \in \Sigma$. Hence, $K$ is a constant function, and the 2 form $\psi^{*} \mathrm{~d} m^{\text {b }}$ can be expressed in the form

$\psi^{*} \mathrm{~d} m^{b}=\frac{1}{M} \varepsilon$

With this result, the integral $\int_{\sigma} \mathrm{d} m^{b}$ can be carried out in the following manner

$\int_{\sigma} \mathrm{d} m^{b}=\int_{\Sigma} \psi^{*} \mathrm{~d} m^{b}=\frac{1}{M} \int_{\Sigma} \varepsilon=\frac{1}{M} \operatorname{area}(\Sigma)$

Remembering that the solid angle $A$ can be expressed as $A=\operatorname{area}(\Sigma) / M^{2}$, we arrive at the equation

$\int_{\sigma} \mathrm{d} m^{b}=M A$

Therefore, the demonstration of phase formula (40) is completed.

In summary, the phase formula (40) is established through a direct application of Stokes' theorem on the integral of differential 1-form $m^{b}$ along the path $\gamma$ defined at the beginning of this appendix (see the Eq. (A8)). The surface integral is directly related to the global geometric property of the distribution of the stress field $\boldsymbol{M}$ along the rod. After careful analysis, we find it could be expressed as the product of the magnitude of the moment and the solid angle enclosed by the closed orbit described by the stress vector $\boldsymbol{M}$.

\section{References}

1. Berry, M.V.: Quantal phase factors accompanying adiabatic changes. Proc. R. Soc. Lond. A 392, 45-57 (1984)

2. Hannay, J.H.: Angle variable holonomy in adiabatic excursion of an integrable hamiltonian. J. Phys. A 18, 221-230 (1985)

3. Berry, M.V.: Classical adiabatic angles and quantal adiabatic phase. J. Phys. A 18, 15-27 (1985)

4. Simon, B.: Holonomy, the quantum adiabatic theorem, and Berry's phase. Phys. Rev. Lett. 51, 2167-2170 (1983)

5. Golin, S., Knauf, A., Marmi, S.: The hannay angles: geometry, adiabaticity, and an example. Commun. Math. phys. 123, 95-122 (1989) 
6. Montgomery, R.: The connection whose holonomy is the classical adiabatic angles of hannay and berry and its generalization to the non-integrable case. Commun. Math. phys. 120, 269-294 (1988)

7. Marsden, J.E.: Lectures on mechanics. Cambridge University Press, Cambridge (1992)

8. Marsden, J.E., Montgomery, R., Ratiu, T.: Reduction, symmetry, and phases in mechanics. Mem. Am. Math. Soc. 88, 1-110 (1990)

9. Love, A.E.H.: A Treatise on the Mathematical Theory of Elasticity, 4th edn. Cambridge University Press, Cambridge (1927)

10. Nizette, M., Goriely, A.: Towards a classification of EulerKirchhoff filaments. J. Math. Phys. 40, 2830-2866 (1999)

11. van der Heijden, G.H.M., Thompson, J.M.T.: Helical and localised buckling in twisted rods: a unified analysis of the symmetric case. Nonlinear Dyn. 21, 71-99 (2000)

12. Montgomery, R.: How much does the rigid body rotate? A Berrys phase from the 18th century. Am. J. Phys. 59, 394-398 (1991)

13. Marsden, J.E., Ratiu, T.: Introduction to Mechanics and Symmetry, 2nd edn. Springer, New York (1999)

14. Simo, J.C.: A finite strain beam formulation. The three-dimensional dynamic problem. Part I. Comput. Methods Appl. Mech. Eng. 49, 55-70 (1985)
15. Simo, J.C., Marsden, J., Krishnaprasad, P.: The Hamiltonian structure of nonlinear elasticity: the material and convective representations of solids, rods, and plates. Arch. Ration. Mech. Anal. 104, 125-183 (1988)

16. Arnol'd, V.I.: Mathematical Methods of Classical Mechanics, 2nd edn. Springer, New York (1989)

17. Spivak, M.: A Comprehensive Introduction to Differential Geometry, Vol. II, 3rd edn. Publish or Perish Inc, Lombard (1999)

18. Marsden, J., Weinstein, A.: Reduction of symplectic manifolds with symmetry. Rep. Math. Phys. 5, 121-130 (1974)

19. Simo, J.C., Wong, K.K.: Unconditionally stable algorithms for rigid body dynamics that exactly preserve energy and momentum. Int. J. Numer. Methods Eng. 31, 19-52 (1991)

20. Landau, L.D., Lifshitz, E.M.: Mechanics, 3rd edn. ButterworthHeinemann, Oxford (1976)

21. Misner, C.W., Thorne, K.S., Wheeler, J.A.: Gravitation. W. H. Freeman and Company, San Francisco (1973) 